\title{
THE COST OF HOVERING AND FORWARD FLIGHT IN A NECTAR-FEEDING BAT, GLOSSOPHAGA SORICINA, ESTIMATED FROM AERODYNAMIC THEORY
}

\author{
ULLA M. NORBERG \\ Department of Zoology, Zoomorphology, University of Gothenburg, \\ Medicinaregatan 18, S-413 90 Gothenburg, Sweden \\ THOMAS H. KUNZ \\ Department of Biology, Boston University, Boston, MA 02215, USA \\ JOHN FLENG STEFFENSEN \\ Marine Biological Laboratory, University of Copenhagen, Strandpromenaden, \\ DK-3000 Helsing $\phi r$, Denmark \\ YORK WINTER AND OTTO VON HELVERSEN \\ Institute of Zoology II, University of Erlangen, Staudtstrasse 5, Erlangen, Germany
}

Accepted 24 May 1993

\begin{abstract}
Summary
Energy expenditure during flight in animals can best be understood and quantified when both theoretical and empirical approaches are used concurrently. This paper examines one of four methods that we have used to estimate the cost of flight in a neotropical nectarfeeding bat Glossophaga soricina (Phyllostomidae), namely the use of kinematic and morphological data and aerodynamic theory to estimate the mechanical power requirements (power output) for hovering and horizontal forward flight. A hot-wire anemometer was used to measure induced velocity (the velocity of air accelerated by the wings) during hovering in order to estimate induced power. Our estimate of aerodynamic power (the sum of induced, profile and parasite powers) required for a $0.0105 \mathrm{~kg}$ G. soricina to hover is $0.15 \mathrm{~W}$ and our estimate of the inertial power (the power required to oscillate the wings) is $0.19 \mathrm{~W}$. Thus, the total mechanical power for hovering is $0.34 \mathrm{~W}$ or $32.4 \mathrm{~W} \mathrm{~kg}^{-1}$. The mechanical power required for horizontal forward flight, near the minimum power flight speed $\left(4.2 \mathrm{~ms}^{-1}\right)$ for a $0.0117 \mathrm{~kg}$ bat is $0.14 \mathrm{~W}\left(12.3 \mathrm{~W} \mathrm{~kg}^{-1}\right)$, of which $0.10 \mathrm{~W}$ is aerodynamic power and $0.042 \mathrm{~W}$ is inertial power. Comparison with our results on metabolic power requirements estimated from nectar intake gives a mechanical efficiency of 0.15 for hovering flight and of 0.11 for forward flight near the minimum power speed.
\end{abstract}

\section{Introduction}

It has long been of interest to estimate how much power is needed to keep an animal

Key words: aerodynamics, bat, Glossophaga soricina, flight energetics, induced velocity, mechanical power. 
flying, from both theoretical and empirical points of view (reviewed by Norberg, 1990). Flying animals have evolved a variety of flight strategies, each requiring different wing designs and energy expenditures. Some species fly continuously during foraging, others perch between foraging bouts and a few hover while foraging. Gliding flight, which requires minimal energy expenditure, is only rarely used by bats. Indeed, nectar-feeding bats frequently hover while foraging, which is one of the most energy-demanding forms of locomotion. Aerial-feeding insectivorous bats perform complex manoeuvres to catch insects and expend considerable energy in doing so.

Aerodynamic theory provides estimates of the mechanical power required to fly (power output) (e.g. Weis-Fogh, 1972, 1973; Pennycuick, 1975, 1989; Norberg, 1976b,c, 1990; Rayner 1979a,b,c; Ellington, 1984c), whereas empirical studies provide estimates of the metabolic energy needed for flight (power input). The metabolic cost of flight depends on the mechanical power requirements and the mechanical efficiency of flight muscles in converting metabolic energy into mechanical work (see, for example, Norberg, 1990, chapter 3). Metabolic power can be estimated directly from wind-tunnel studies (Tucker, 1968, 1972; Thomas, 1975; Carpenter, 1975, 1985, 1986), from field studies using doubly labelled water or from balance methods using estimates of metabolic energy consumed (von Helversen and Reyer, 1984; Masman and Klaassen, 1987; Kunz and Nagy, 1988; Nagy, 1989; Winter et al. 1993).

One approach towards understanding animal flight is to apply theories derived from studies of aircraft wings and propellers. However, aerodynamic theory contains approximations, simplifications and empirical coefficients, and the situation is especially complicated for a flapping wing, in which angles, velocities and shape change continuously. To derive estimates of power input of flying animals from aerodynamic theory requires assumptions about the mechanical efficiency of muscle in converting fuel to mechanical work. Mechanical efficiency depends on the size of the animal (Heglund et al. 1982) and on flight speed (cf. data on swimming speeds in fish in Webb, 1971) and is rather laborious to estimate. Only a few physiologists (Tucker, 1968, 1972; Thomas, 1975; Bernstein et al. 1973; Hudson and Bernstein, 1983) have estimated the mechanical efficiency of flying animals (two bat and four bird species) at different speeds by measuring $\dot{V}_{\mathrm{O}_{2}}$ in slightly tilted wind tunnels. No realistic regression equation of mechanical efficiency versus body mass (or speed) is available for bats and, therefore, results from aerodynamic theory cannot reliably be used to estimate the metabolic power input during flight. Nevertheless, aerodynamic theory is an invaluable tool for developing our understanding of the connection between flight morphology, flight mode and flight behaviour, including any modelling of how flight costs vary with changes in the morphology of the animal.

Power input to flying animals can be measured using various empirical methods but, like theoretical approaches, each method has its advantages and disadvantages. The most direct way to estimate flight costs is to measure oxygen consumption and then to convert the amount of oxygen consumed into estimates of energy expenditure. To do this, one must make assumptions regarding the amount of energy released when a food substrate is oxidized. Most empirical estimates of flight metabolism refer to measurements of the total amount of energy consumed while the animal performs short flights at various 
speeds, including occasional manoeuvres. The calculation of power output from such results involves several assumptions even under the most favourable circumstances, and this approach cannot be used for comparison with predictions from mechanical theory (Pennycuick, 1989).

Field data on time budgets and estimates of metabolism derived from doubly labelled water (DLW) studies provide estimates of power ouput. These methods also include errors, and one should expect both intraspecific and interspecific variation in flight costs. Flight in free-ranging animals is seldom straight and horizontal. Turns and manoeuvres are characteristic of many aerial-feeding insectivorous bats (Norberg, 1976a; Rayner and Aldridge, 1985) and others that fly in cluttered environments (Norberg and Rayner, 1987). In addition, wing loading (body weight/wing area) may vary between individuals; in pregnant female bats it can increase by as much as 50\% compared with non-pregnant bats (when foetus and food load are considered) (Kunz, 1974; Anthony and Kunz, 1977). Hughes and Rayner (1991) artificially increased wing loading in the long-eared bat (Plecotus auritus) and found that flight speed fell and wingbeat frequency increased with total mass and wing loading, and they calculated that a 50\% increase in wing loading increased power requirements by about $140 \%$.

In the present study, we used independent methods for estimating the energy costs of hovering and horizontal forward flight of a neotropical nectar-feeding bat Glossophaga soricina (Glossophaginae, Phyllostomidae), tested in the laboratory. These estimates were based on (1) kinematic and morphological data and aerodynamic theory, (2) energy intake of nectar, (3) $\mathrm{O}_{2}$ consumption from respiration measurements (hovering costs only), and (4) $\mathrm{CO}_{2}$ production estimated with the doubly labelled water technique, where methods 2 and 4 were each combined with time budgets. We used these approaches to compare the different methods for estimating flight costs in a flying vertebrate. This paper estimates the mechanical power (power output) requirements for hovering and horizontal forward flight based on aerodynamic theory. Results from method 2 appear in Winter et al. (1993) and results from methods 3 and 4 are in preparation.

\section{Materials and methods}

This study was conducted at the Department of Zoology in Erlangen, Germany. Our study was based on G. soricina antillarum (Rehn) collected in Jamaica and maintained in captivity. Individual bats were trained to fly in flight cages and to hover in front of a feeder while feeding on nectar. Two individuals were used for the aerodynamic analyses of hovering and one individual for forward flight. The body mass varied among the individuals and during the day. The masses used (see Table 1) are mean values for the bats used in the hovering and forward flight studies. They were derived from computerstored data from the periods during which the flight kinematic data were collected. The wing spans were the same in all three individuals. The wing area was estimated in one of the bats and assumed to be the same in the others.

The flight chamber used for the hovering and respiration experiments was constructed as a wooden frame with solid pressed-wood ends ( $4 \mathrm{~m}$ long, $1 \mathrm{~m}$ high and $1.26 \mathrm{~m}$ wide). The top and sides were covered with polyethylene plastic foil and the bottom with plastic- 
coated wire netting; the distance from the bottom of the cage to the solid floor was $1 \mathrm{~m}$. The flight tunnel used for horizontal flight studies was U-shaped (total length $14 \mathrm{~m}$, height $2 \mathrm{~m}$ and width $1 \mathrm{~m}$ ) and was covered with plastic film. A cork suspended from a microbalance provided the only roosting place between flights in the two cages.

Wingbeat kinematics (wingbeat frequency and amplitude, inclination of the body and stroke plane, and the wing positions during the wingbeat) of the bats were recorded by a Photosonics high-speed film camera (200 frames ${ }^{-1}$ ) with an Angenieux zoom lens 12-120 mm, f2.0, and a Sony Handycam High 8 video, and still photographs were taken with a Leicaflex SL equipped with a Sumicron $50 \mathrm{~mm}$, f2.0 lens. The hovering sequences were recorded about $0.3-0.4 \mathrm{~m}$ from one end wall, where the feeder was mounted. The distances to the other walls, the ceiling and the net bottom of the chamber were about $0.5 \mathrm{~m}$, and the distance to the solid floor was about $1.5 \mathrm{~m}$. Flight speeds were obtained from the slow-motion films by estimating the number of body lengths travelled per seconds. A string grid, forming $2 \mathrm{~cm} \times 2 \mathrm{~cm}$ squares, was used as a background scale, corrected for the distance between the grid and the bat. SI units are used throughout this study.

\section{Aerodynamic theory}

Animal wings act as aerofoils and a flying animal must generate a momentum flow to maintain lift. For bats and birds, this momentum of the air is only transported as a vortex. The wings generate weight support and thrust owing to the presence of this shed vortex across the wing. The essence of hovering flight is the production of a vertical force which must balance the animal's weight $(\boldsymbol{M g}=$ mass times acceleration of gravity, measured in $\mathrm{N})$. Hovering flapping flight is more expensive than forward flight because it involves no forward speed component. This means that the surface area through which air is accelerated during a unit of time is much smaller than that for forward flight. Hence, less air must be accelerated to a higher velocity and, since power equals the air mass times the square of the downward induced velocity, this requires more power.

Hummingbirds and several species of insects use normal hovering, which is defined by Weis-Fogh $(1972,1973)$ as active flight on the spot with the wings moving through a large angle in an approximately horizontal plane in a figure-of-eight motion with symmetrical half-strokes. In hovering bats and other hovering birds, the stroke plane is usually more tilted, and the wings are flexed during the upstroke to avoid large drag forces and negative (downwardly directed) lift forces. In this asymmetrical hovering the upstroke is a recovery stroke, giving zero or slight negative lift, so that all useful lift must be produced during the downstroke. In both normal and asymmetrical hovering, the wing strokes produce periodic pressure pulses, one for each wing stroke in asymmetrical hovering and two for each wing stroke in normal hovering, so the wake consists of a chain of vortex rings.

The flight muscles of a flying animal do mechanical work when the point of application of a force is moved. The work is the distance moved times the force component in the direction of motion, and the rate at which this work is done is the mechanical power. Because drag is caused by several factors, the aerodynamic power includes three different components: induced power $\left(P_{\text {ind }}\right)$, the rate of working required to generate a vortex wake whose reaction generates lift and thrust, profile power $\left(P_{\mathrm{pro}}\right)$, the work needed to 
counteract form and friction drag of the wings, and parasite power $\left(P_{\mathrm{par}}\right)$, the work needed to counteract form and friction drag of the body. Inertial power $\left(P_{\text {iner }}\right)$, the work needed to accelerate the wings at each stroke, must also be considered.

The mechanical power required to fly is the sum of these four components, and the corresponding metabolic power for flight is the mechanical power divided by the mechanical efficiency $(\eta)$ plus the basal metabolic rate (BMR). Furthermore, Tucker (1973) estimated that the extra amounts of mechanical power required during flight to circulate the blood and to ventilate the lungs were each about $5 \%$ of the total power required for other purposes. Therefore, the metabolic power for flight should be multiplied by a circulation and ventilation factor of 1.1 (10\% addition). The power input then becomes:

$$
P_{\text {met }}=1.1\left\{\left[\left(P_{\text {ind }}+P_{\text {pro }}+P_{\text {par }}+P_{\text {iner }}\right) / \eta\right]+\text { BMR }\right\},
$$

expressed in watts (W) (Pennycuick, 1975, see also Norberg, 1990). In hovering flight, parasite power is taken to be zero, since there is no forward speed component and the effect of the induced velocity is negligible.

Unsteady mechanisms may be utilized to generate the requisite forces for weight support. The aerodynamic theory employed here is quasi-steady. If unsteady effects are involved, a quasi-steady approach may underestimate the induced power, but the other power components are not affected. Rayner (1979c) and Ellington (1984c) applied vortex theory to the calculation of induced power and provided correction coefficients for estimates based on quasi-steady analyses. We have derived this coefficient by using empirical measurements of the induced velocity (see below).

\section{Hovering flight}

Induced power. The induced power is the principal power component in hovering and slow flight. A hovering animal beating its wings horizontally in symmetrical hovering flight can be compared to a helicopter whose rotors drive the air downwards through the disk formed by the sweeping rotor blades. The airflow through the disk is due entirely to the induced velocity $w$ (Fig. 1), which is assumed to be uniform over the whole disk area. According to the Rankine-Froude axial momentum-jet theory, the air accelerates to the velocity $2 w$ far below the animal (approximately one wing span) and the induced power is the product of the weight of the animal (mass times acceleration $\boldsymbol{g}$ of gravity, $\boldsymbol{M g}$ ) (here equivalent to the induced drag) and the induced velocity $w$ :

$$
P_{\text {ind, RF }}=M \boldsymbol{g} w=(M g)^{3 / 2} /\left(2 \rho S_{\mathrm{d}}\right)^{1 / 2},
$$

where $\rho$ is air density and $S_{\mathrm{d}}=\pi b^{2} / 4$ and is the disk area (the area of a circle with wing span $b$ as diameter; Fig. 1). This gives a minimum value of the induced power. For hovering animals, the wake consists of periodic pulses and the actual induced power therefore includes the dimensionless induced factor $k$, and is expressed as:

$$
P_{\text {ind }}=k M g w .
$$

The value of $k$ has been assumed by Pennycuick $(1975,1989)$ to be 1.2 for animal wings, and it has been estimated using the vortex theory by Rayner (1979c, equation 13) and 
A

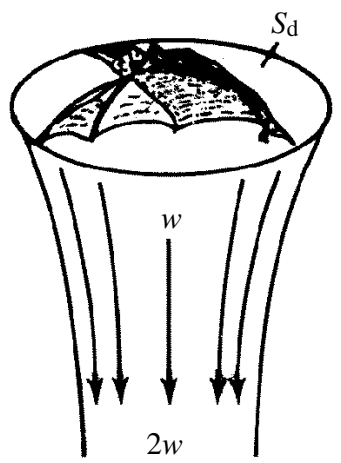

B
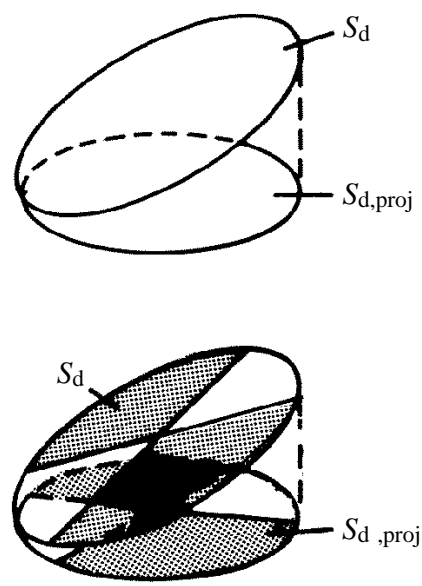

Fig. 1. (A) Model of a continuous momentum jet past a hovering animal. Air is sucked down from rest far above the animal, passes through the wing disk at the induced velocity $w$ and reaches twice that velocity farther down in the most contracted zone, according to the momentum-jet theory. In animals, however, the wake is periodic because the wings do not act as propellers. (B) Areas used by different authors to estimate induced velocity from the momentum theory. $S_{\mathrm{d}}$ is the disk area, which is the area of a circle with wing span as diameter, and $S_{\mathrm{d} \text {,proj }}$ is the projection of the disk area on the horizontal plane. $S_{\mathrm{d}}{ }^{\prime}$ is the area swept by the wings and $S_{\mathrm{d}}{ }^{\prime}$,proj is its projection.

Ellington (1984b, equation 48; 1984c, equation 3). In Rayner's and Ellington's theories, the wake is modelled by a chain of co-axial, small-cored, circular vortex rings stacked one upon another, each member being generated by a single wing stroke. Ellington (1984c) estimated the values of $k$ for hovering insects with a horizontal stroke plane to be 1.11-1.21 and for insects with an inclined stroke plane to be 1.57-1.65.

The velocity of the airstream (induced velocity) accelerated downwards by the wings below the bat during hovering can also be measured empirically. We used a hot-wire anemometer (developed at the Max-Planck Institute, Seewiesen) to determine the downward velocity at different locations below the bat (Fig. 2). The anemometer measured total air velocity and gave measurements in voltage. It was calibrated to corresponding air velocity at the Institut für Strömungsmechanik, University of Erlangen (Professor F. Durst). The theoretically estimated velocity, based on a constant jet stream, was compared with the empirically measured value to derive the corresponding induced factor $k$. It is important also to test whether the entire disk area $S_{\mathrm{d}}$ should be used in equation 2. Should the area of the projection of this disk, of the swept sectors or of the projection of the swept sectors be used instead (cf. Ellington 1984b,c; Fig. 1)? If one of the smaller areas is used, the corresponding $k$ value will be lower than when the whole disk is used.

Profile power. The profile power increases with wing area (wing length and width) and with increasing speed. Rayner $(1979 b, c)$ calculated this power component for asymmetrical hovering by a method derived from Weis-Fogh (1972), as:

$$
P_{\text {pro }}=0.0166 \mu \rho b^{3} S C_{\mathrm{D}, \mathrm{pro}} \theta / \tau^{2} T^{3},
$$




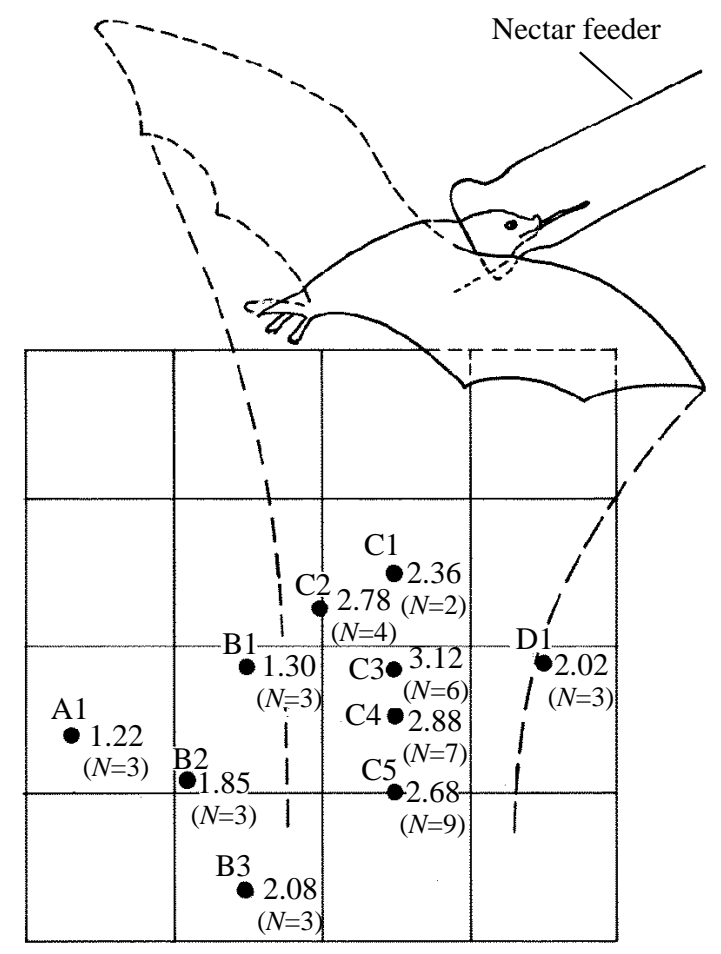

Fig. 2. Positions of the hot-wire anemometer located below the hovering bat (filled circles; $\mathrm{A}-\mathrm{D}$ ) and the average downward air velocities (in $\mathrm{m} \mathrm{s}^{-1}$ ) measured at these sites. Outside and below these positions, the air was either turbulent or calm. The most stable conditions occurred in the core of the airflow at positions $\mathrm{C} 1-\mathrm{C} 5$, which are outlined with dashed lines. $N$ gives the number of hovering bouts, each of which contained 9-30 wing strokes. The sides of the squares in the grid behind the bat are $10 \mathrm{~cm}$ long, which corresponds to $5.5 \mathrm{~cm}$ in the plane of the bat.

where $\mu$ is the muscle ratio, $S$ is wing area, $C_{\mathrm{D} \text {,pro }}$ is profile drag coefficient, $\theta$ is wingstroke amplitude and $\tau$ is the downstroke ratio (the time the downstroke takes in proportion to the time $T$ for the entire wing stroke). The muscle ratio $\mu$ is defined as $\left(m_{\mathrm{s}}+m_{\mathrm{p}}\right) / m_{\mathrm{p}}$, where $m_{\mathrm{s}}$ is the mass of the muscles powering the upstroke and $m_{\mathrm{p}}$ the mass of those powering the downstroke. In Plecotus auritus which, like G. soricina, also sometimes hovers, the muscle ratio is 1.2 (U. M. Norberg, unpublished results). This value was adopted in the present study. The drag coefficient $C_{\mathrm{D} \text {,pro }}$ depends on the shape and smoothness of the wing surface, the boundary layer condition and the Reynolds number (see Norberg, 1990) and is taken to be constant for the wings and equal to 0.02 (Rayner, 1979b; Pennycuick et al. 1992).

Inertial power. The inertial power is considered to be low during fast forward flight, since wing inertia can be converted into useful aerodynamic work at the bottom of the downstroke (Pennycuick, 1968; Norberg, 1976b), but it is higher during hovering and slow flight. The significance of inertial power has been intensively investigated by WeisFogh $(1972,1973)$. In medium and fast forward flight, work must be done to accelerate 
the wings at the beginning of the downstroke, but at the end of the downstroke they can be decelerated and reversed by aerodynamic forces, the inertial forces thus being converted into useful aerodynamic forces. One uncertainty is the extent to which inertial power is in fact converted in this way into useful aerodynamic power. In slow and hovering flight, this transfer of energy is not as easily achieved because the relative airspeed at the turning points is very low as a result of the small or absent forward speed component. The loss of inertial power would therefore be important, unless kinetic energy can be removed and restored by some other means. The primary feathers in birds and the elastic and flexible bat wing might increase the efficiency of transfer of the wing's kinetic energy to the air towards the end of the downstroke (Pennycuick and Lock, 1976; Norberg, 1990). At the end of the upstroke, the downstroke muscles must brake the movement and absorb the excess kinetic energy of the wings. The angular momentum of the wings thus causes a stretching of the downstroke muscles, which they actively resist. Work done during stretching a muscle is termed negative work. Negative work costs only about $20 \%$ (in metabolic energy) of the same amount of positive work (at least in human locomotion; Margaria, 1968). Following Weis-Fogh (1972, p. 91), the negative work is disregarded here so as not to exaggerate the estimate of inertial work and power.

If $I$ is the moment of inertia of the wings' masses about the fulcra (shoulder joints), the acceleration of the wings give rise to an inertial bending moment $Q$, which equals $I$ times the angular acceleration of the wings. Assuming that the wings oscillate in simple harmonic motion (which is the case in Plecotus auritus, Norberg 1976b,c), the bending moment is:

$$
Q(t)=-2 I \pi^{2} f_{\mathrm{w}}^{2} \theta \sin \left(2 \pi f_{\mathrm{w}} t\right)
$$

(Weis-Fogh, 1972, 1973), where $t$ is time. $Q$ varies linearly with the positional angle $\gamma$ of the long axis of the wing in the stroke plane; it is maximal at the extreme wing positions and zero at the middle of the stroke. To calculate the inertial work, we must integrate $Q$ over a complete wing stroke (see Weis-Fogh, 1972, for procedure). The total inertial work during one wing stroke is the sum of the work done during the upstroke and during the downstroke, and the inertial power is this work times the wingstroke frequency:

$$
P_{\text {iner }}=f_{\mathrm{w}}\left[\int_{\gamma_{\min }}^{\gamma_{\max }} Q \mathrm{~d} \gamma+\int_{\gamma_{\max }}^{\gamma_{\min }} Q \mathrm{~d} \gamma\right] .
$$

To estimate the moment of inertia, $I$, we used the multiple regression derived by Thollesson and Norberg (1991) for bats and two wings:

$$
I=8.98 \times 10^{-3} M^{0.53} b^{2.15} S^{0.65} .
$$

Kinetic energy is to some extent also being converted into useful aerodynamic work in hovering and slow flight, so that the true expense becomes somewhat reduced (WeisFogh, 1972). In the hummingbird Amazilia fimbriata (mass $0.005 \mathrm{~kg}, f_{\mathrm{w}} 35 \mathrm{~s}^{-1}$ ), the estimated reduction of total mechanical power (the sum of the aerodynamic and the 
inertial power) during hovering flight was about $18 \%$ (Weis-Fogh, 1972) and in the longeared bat Plecotus auritus (mass $0.009 \mathrm{~kg}, f_{\mathrm{w}} 11.9 \mathrm{~s}^{-1}$ ) in slow forward flight the reduction was about 14\% (Norberg, 1976b). To determine how much of the total mechanical work is wasted, i.e. how much of the inertial work is not converted into useful aerodynamic work, we used Weis-Fogh's (1972) aerodynamic model, in addition to the one given above. Weis-Fogh made quantitative estimates of force, work and power with the blade-element theory, using a compound drag coefficient to lump together the aerodynamic power components. We calculated the aerodynamic and inertial torques for three different wing segments and for 14 different wing positions. The torque which the wing-stroke muscles have to produce is then obtained by adding the aerodynamic torque and the inertial torque for every wing position separately. Since inertial torque changes sign during the wing stroke, this sum becomes less than when adding the total aerodynamic torque and the total inertial torque. The difference is due to the amount of inertial work that is converted into useful aerodynamic work. This difference was then subtracted from the inertial power obtained from equation 6 to estimate the true costs due to wing inertia.

For small flying animals (insects), the viscous forces are large and the inertia of the wings will be increased by the mass of air attaching to them and that is accelerated along with them (wing virtual mass), leading to an apparent increase in wing mass (Ellington, $1984 a, c)$. The virtual mass of the wing pair was taken by Ellington $(1984, a, c)$ to be proportional to the mass of air in a cylinder of length equal to the wing span and with a diameter equal to the mean wing chord. We consider wing virtual mass to be unimportant for animals the size of bats and birds, since viscous forces at the high Reynolds number in question are very small, and it is disregarded here. The possible effect of it is, however, discussed. The Reynolds $(R e)$ number is the ratio of inertial force to frictional force and is expressed as length of the body times speed divided by the kinematic viscosity of the fluid.

\section{Horizontal forward flight}

Induced power. The induced power during forward flight equals $M g w$, as in hovering flight, but the induced velocity is smaller. In forward horizontal flight it can be expressed as:

$$
P_{\text {ind }}=M \boldsymbol{g} w=k(M g)^{2} / 2 \rho S_{\mathrm{d}} V,
$$

where $V$ is forward flight speed (e.g. Pennycuick, 1968, 1975; Norberg, 1990). We used the value of $k$ obtained from our measurements with the hot-wire anemometer of the induced velocity in hovering flight, although the bats in the horizontal flight study were somewhat heavier $(0.0117 \mathrm{~kg})$ than those used in the anemometer experiments $(0.0105 \mathrm{~kg})$. This may be a reasonable approximation, since the coefficient $k$ should increase with increased weight of the animal and with decreased flapping frequency and stroke amplitude, but should be lower in forward flight than in hovering owing to smaller variations in the induced velocity during the wing stroke.

Profile power. The profile power can be found by integration over the whole wings during a complete wing stroke. The wing was divided into three strips, and the velocities 
and forces were estimated at the middle of these strips (at $r=0.16 l_{\mathrm{w}}, 0.49 l_{\mathrm{w}}$ and $0.82 l_{\mathrm{W}}$ from the wing base). The profile power is then:

$$
P_{\text {pro }}=(1 / 2) \rho C_{\mathrm{D}, \text { pro }} \int_{t=0}^{t=T} \sum_{r=1}^{r=3} S(r) V_{\mathrm{R}}(r, t)^{3} \mathrm{~d} t .
$$

The flapping velocity fluctuations were estimated by assuming harmonic oscillation (Weis-Fogh, 1972, 1973; Norberg, 1976b). The procedures used for calculating resultant air velocities $\left(V_{\mathrm{R}}\right.$ at each strip and wing position are given in Norberg (1976b). A value of 0.02 was used for $C_{\mathrm{D} \text {,pro. }}$.

Parasite power. Parasite power varies with forward flight speed $V$ as:

$$
P_{\mathrm{par}}=(1 / 2) \rho V^{3} S_{\mathrm{b}} C_{\mathrm{D}, \mathrm{par}},
$$

where $S_{\mathrm{b}}$ is the frontal projected area of the body and $C_{\mathrm{D} \text {,par }}$ is the coefficient of parasite drag. To estimate the body frontal area $S_{\mathrm{b}}$ we used the regression of Pennycuick et al. (1988), $S_{\mathrm{b}}=\left(8.13 \times 10^{-3}\right) M^{2 / 3}$, based on frozen birds in wind tunnel measurements, and we used a value of $C_{\mathrm{D} \text {,par }}$ of 0.40 , suggested by the authors for bird bodies at Reynolds number below 50000 .

Inertial power. The inertial power was estimated as for hovering flight.

\section{Results}

Morphological measurements and flight data for hovering and forward flight are given in Table 1. It was difficult to obtain exact data on wingbeat amplitudes and stroke-plane angles but small deviations in these angles do not have any large effect on the calculations; these angles were measured from a few representative flights and wing

Table 1. Morphological data and flight variables of Glossophaga soricina used in calculations in the text

\begin{tabular}{lll}
\hline & Hovering & Forward flight \\
\hline Body mass, $M$ & $0.0105 \mathrm{~kg}$ & $0.0117 \mathrm{~kg}$ \\
Body weight, $M g$ & $0.103 \mathrm{~N}$ & $0.115 \mathrm{~N}$ \\
Wing length, $l_{\mathrm{w}}$ & $0.117 \mathrm{~m}$ & $0.117 \mathrm{~m}$ \\
Wing span, $b$ & $0.258 \mathrm{~m}$ & $0.258 \mathrm{~m}$ \\
Wing area, $S$ & $0.0107 \mathrm{~m}^{2}$ & $0.0107 \mathrm{~m}^{2}$ \\
Wing-disk area, $S_{\mathrm{d}}$ & $0.0523 \mathrm{~m}^{2}$ & $0.0523 \mathrm{~m}^{2}$ \\
Forward speed, $V$ & $0 \mathrm{~m} \mathrm{~s}^{-1}$ & $4.2 \mathrm{~m} \mathrm{~s}^{-1}$ \\
Induced velocity, $w$ & $1.43 \mathrm{~m} \mathrm{~s}^{-1}$ & - \\
Wing-stroke amplitude, $\theta$ & $120^{\circ}(2.09 \mathrm{rad})$ & $88^{\circ}(1.54 \mathrm{rad})$ \\
Stroke-plane angle, $\alpha$ & $30^{\circ}$ & $54^{\circ}$ \\
Wingbeat frequency, $f_{\mathrm{w}}$ & $15.20 \mathrm{~s}^{-1}$ & $11.76 \mathrm{~s}^{-1}$ \\
Wingbeat duration, $T=f_{\mathrm{w}}{ }^{-1}$ & 0.0658 & 0.0850 \\
Downstroke ratio, $\tau$ & 0.48 & 0.50 \\
Air density, $\rho$ & $1.177 \mathrm{~kg} \mathrm{~m}^{-3}$ & $1.177 \mathrm{~kg} \mathrm{~m}^{-3}$ \\
\hline
\end{tabular}


strokes from the high-speed films, which were taken from the side and from below the flying bat. In hovering flight, wingbeat frequency was 15.2 strokes s$^{-1}$ (mean of 23 hovering bouts including 286 wing strokes, S.D. $=0.346$ strokes s $^{-1}$ ), wing-stroke amplitude was about $120^{\circ}$ and stroke-plane angle (the inclination of the stroke plane to the horizontal plane) about $30^{\circ}$. von Helversen (1986) measured wingbeat frequency in a hovering G. soricina (body mass $0.0101 \mathrm{~kg}$ ) to be $16 \mathrm{~Hz}$ and wingbeat amplitude to be $120^{\circ}$, as in our experiment. In the present study, the measured downstroke ratio in hovering flight was $0.48(N=51)$, but we used 0.5 to simplify the calculation for inertial power. In forward flight, wing-stroke amplitude was about $88^{\circ}$, stroke-plane angle about $54^{\circ}$ and wingbeat frequency 11.8 strokes s$^{-1}$ (measured from one representative wing stroke). Downstroke ratio in forward flight was difficult to obtain from the films but was close to, and taken to be, 0.5. The forward flight speed was $4.2 \mathrm{~ms}^{-1}$.

\section{Induced velocity}

The positions of the hot-wire anemometer during hovering and the corresponding velocities of the airstream caused by the beating wings during hovering are shown in Fig. 2. We measured the velocity at a large number of positions both outside and below these positions to determine the spatial variation. The air just outside positions B and D was somewhat unstable and the air velocity gradually decreased to zero farther out. The velocities at C2-C5 (Fig. 2) are assumed to be twice the velocity at the wing disk (that is, twice the induced velocity) in accordance with the momentum theory. However, the velocity oscillates with the wingbeat during a hovering bout (Fig. 3). The value of $2 w$ $\left(2.85 \mathrm{~ms}^{-1}\right)$ used in our study represents the mean of 22 hovering bouts from one bat (C3-C5) and four from another (C2), with each sample based on 9-30 wingbeats. $2.85 \mathrm{~m} \mathrm{~s}^{-1}$ is the grand mean value of the mean of 466 maximum values (representing the velocity of the airstream produced by downstrokes, mean $3.39 \mathrm{~ms}^{-1}$, S.D. $0.249 \mathrm{~ms}^{-1}$ ) and the mean of 446 minimum values (representing the velocity of the airstream produced by upstrokes, mean $2.31 \mathrm{~ms}^{-1}$, S.D. $0.263 \mathrm{~ms}^{-1}$ ).

\section{Power required for hovering}

The estimated power components for hovering flight in G. soricina (body mass $0.0105 \mathrm{~kg}$ ) are given in Table 2.

\section{Induced power}

Theoretically, the Rankine-Froude (momentum-jet) value of the minimum induced power for G. soricina is $P_{\text {ind, RF }}=0.094 \mathrm{~W}$ (right-hand part of equation 2). Using our empirically derived average value of the induced velocity instead of the theoretically estimated velocity, the power becomes $P_{\text {ind }}=M g w=0.147 \mathrm{~W}$. This corresponds to $k=1.56$ in equation 3 , when the entire disk area, $S_{\mathrm{d}}$, is used. This value lies close to that obtained using Ellington's $(1984 b, c)$ theoretical model $(k=1.53)$ for $G$. soricina, and it is $10 \%$ higher than that predicted from Rayner's (1979c) model ( $k=1.42)$. The corresponding $k$

values when using the projected area of the wing disk $\left(S_{\mathrm{d}, \text { proj }}=S_{\mathrm{d}} \cos 30^{\circ}\right.$, Fig. 1), the area of the sectors swept by the wings $\left[S_{\mathrm{d}^{\prime}}=(2 / 3) S_{\mathrm{d}}\right]$ or the projection of this area $\left(S_{\mathrm{d}^{\prime}}\right.$,proj $)$ are 


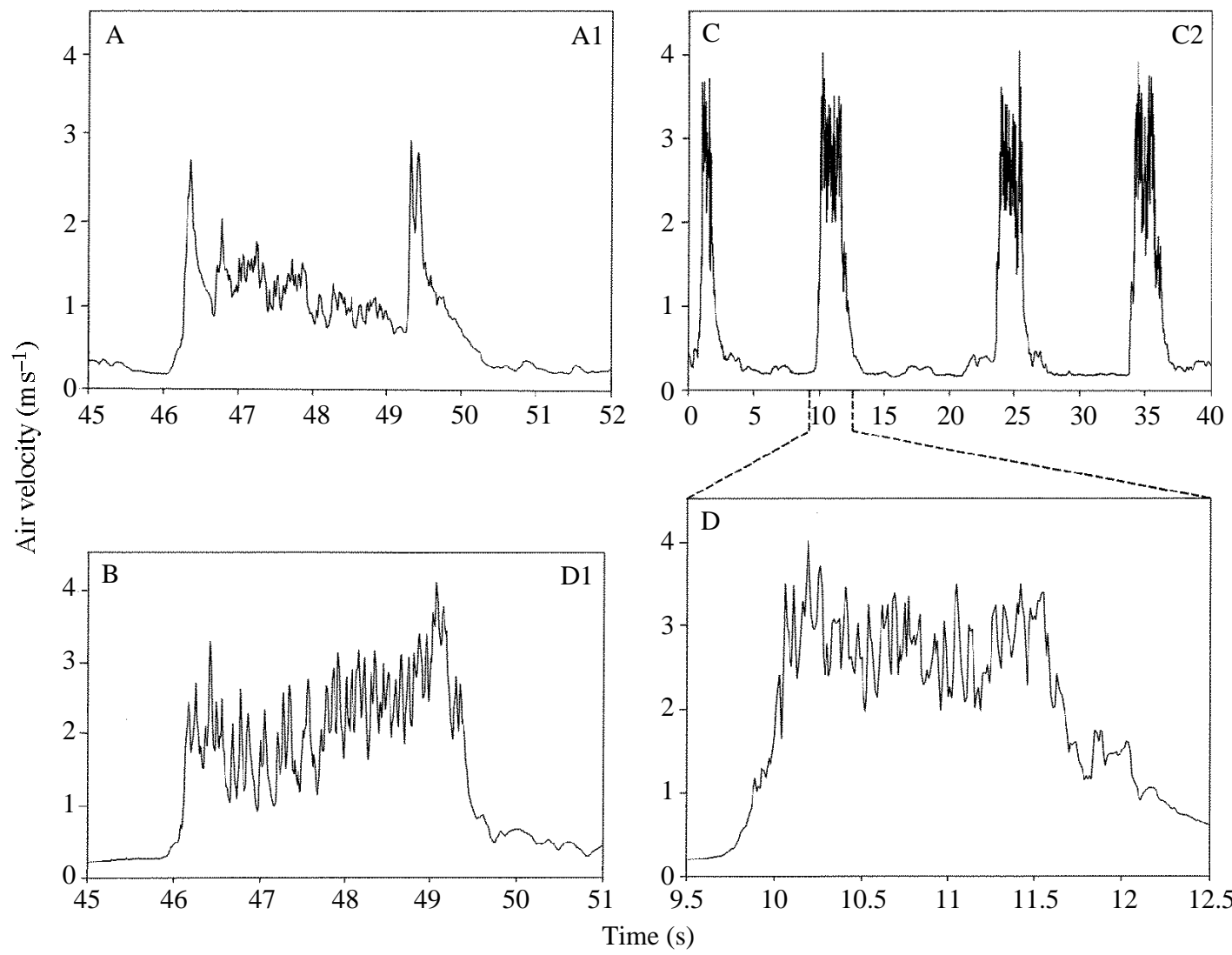

Fig. 3. Downward air velocity plotted against time during hovering in Glossophaga soricina at the positions (A) A1, (B) D1 and (C,D) C2 shown in Fig. 2. A and B each show one selected hovering bout. $\mathrm{C}$ shows several hovering bouts and $\mathrm{D}$ shows an expansion of one selected bout. The velocity oscillates over the wing stroke, with one peak per stroke; maximum values are produced by downstrokes and minimum values by upstrokes, and the wingbeat frequency can thus be estimated from the number of peaks.

$1.44,1.28$ and 1.18, respectively. The use of any of these areas in equation 2 would thus probably underestimate the induced power in hovering flight in bats.

\section{Profile power}

Profile power is estimated to be $P_{\text {pro }}=0.0058 \mathrm{~W}$.

\section{Inertial power}

The moment of inertia during the downstroke for both wings is $I=2.28 \times 10^{-6} \mathrm{~kg} \mathrm{~m}^{2}$. During the upstroke, the wings of $G$. soricina are flexed by approximately $30 \%$, thereby reducing the moment of inertia by $50 \%$ (to $1.14 \times 10^{-6} \mathrm{~kg} \mathrm{~m}^{2}$ ). Fig. 4 shows the inertial torque (bending moment) as a function of the positional angle $\gamma$ of the wings. The inertial torque changes sign at the middle of the stroke and, since the oscillation is assumed to be 
Table 2. Estimated values of the mechanical power required for hovering and horizontal flight (at $4.2 \mathrm{~ms}^{-1}$ ) of the nectar-feeding bat Glossophaga soricina of body mass $0.0105 \mathrm{~kg}$ and $0.0117 \mathrm{~kg}$, respectively

\begin{tabular}{llc}
\hline Power & Hovering & Forward flight \\
\hline Induced power, $P_{\text {ind }}$ & $0.15 \mathrm{~W}$ & $0.040 \mathrm{~W}$ \\
Profile power, $P_{\text {pro }}$ & $0.0058 \mathrm{~W}$ & $0.055 \mathrm{~W}$ \\
Parasite power, $P_{\text {par }}$ & $0 \mathrm{~W}$ & $0.0068 \mathrm{~W}$ \\
Inertial power, $P_{\text {iner }}$ & $0.26 \mathrm{~W}$ & $0.069 \mathrm{~W}$ \\
True inertial power, $P_{\text {iner,true }}$ & $0.19 \mathrm{~W}$ & $0.042 \mathrm{~W}$ \\
Summed mechanical power, $P_{\text {hov }}$ or $P_{\text {hor }}$ & $0.34 \mathrm{~W}$ & $0.14 \mathrm{~W}$
\end{tabular}

The total mechanical power is $P_{\text {hov }}\left(\right.$ or $\left.P_{\text {hor }}\right)=P_{\text {ind }}+P_{\text {pro }}+P_{\text {par }}+P_{\text {iner,true }}$.

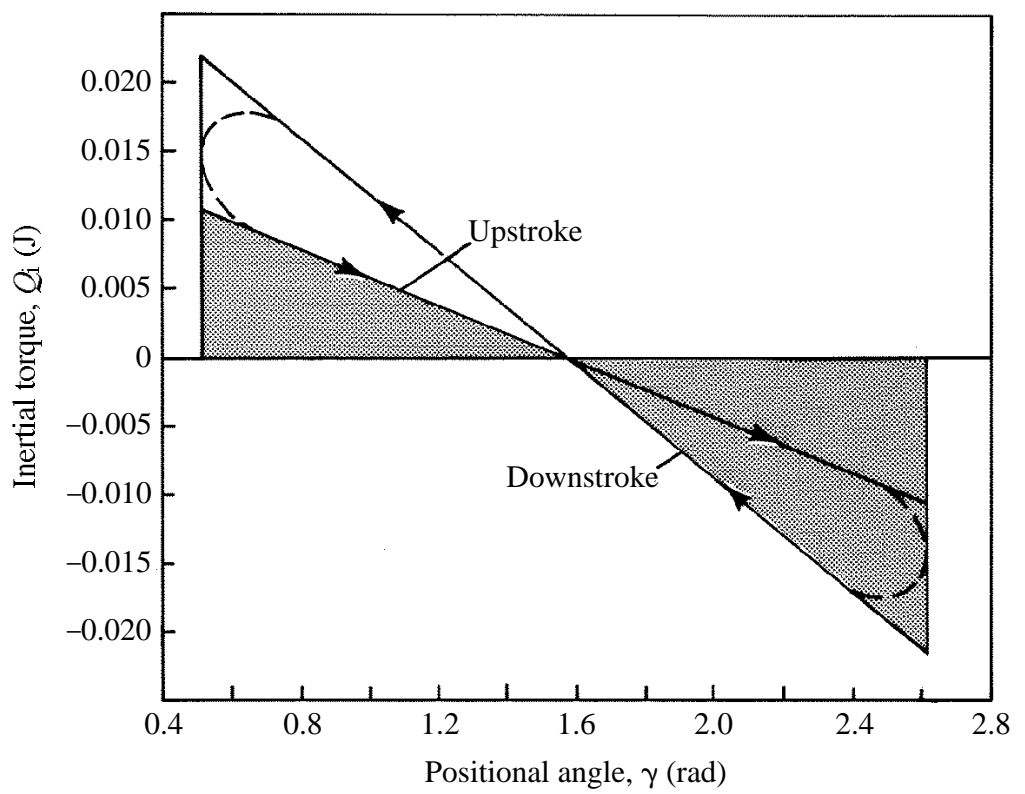

Fig. 4. The variation of the inertial torque $\left(Q_{\mathrm{i}}\right)$ during a complete wing stroke of Glossophaga soricina during hovering flight when the wingbeat frequency, $f_{\mathrm{w}}$, is $15.2 \mathrm{~s}^{-1}$. The work done during the first half of the downstroke is represented by the shaded area to the right, and the work done during the first half of the upstroke is represented by the shaded area to the left. The inertial power is this work times the flapping frequency. Since the flapping velocity of the wings is not zero at the turning points, the transition between the downstroke and upstroke curves should be rounded (as indicated by the dashed lines). This does not have any significant effect on the calculations, since the additional work during the upstroke and the saved work during the downstroke almost cancel each other out.

harmonic, it varies linearly with the positional angle. A positive sign indicates that the force is directed in the morphologically dorsal direction, and a negative sign that the force is directed in the morphologically ventral direction. The inertial torque integrated over a 
quarter of a wing stroke is the work done in accelerating the wing pair up to its maximum angular velocity during the first half of each half-stroke. It is $0.0115 \mathrm{~J}$ during the downstroke and $0.0057 \mathrm{~J}$ during the upstroke. The total inertial power is $f_{\mathrm{w}}$ times this work and is $P_{\text {iner }}=15.2(0.0115+0.0057)=0.261 \mathrm{~W}$.

The total aerodynamic and inertial power required for $G$. soricina to hover is $P_{\text {hov }}=P_{\text {ind }}+P_{\text {pro }}+P_{\text {iner }}=0.414 \mathrm{~W}$. To determine how much of the kinetic energy is converted into useful aerodynamic work, we followed the procedure of Weis-Fogh (1972). The total torque and work which the muscles have to produce is found by adding the aerodynamic and inertial torque components for every wing position separately; see Weis-Fogh (1972, p. 90 ff.) and Fig. 6 for the procedure for estimating inertial power for $G$. soricina in horizontal forward flight. The true expense then becomes $P_{\text {hov }}=0.340 \mathrm{~W}$, which is a reduction of about $18 \%$, or $0.074 \mathrm{~W}$, of the total mechanical power. About $28 \%(0.074 \mathrm{~W})$ of the inertial work and power is thus converted into useful aerodynamic work and power, whereas $0.187 \mathrm{~W}(72 \%$; the true inertial power, $P$ iner,true) is wasted.

\section{Resting and basal metabolic rate}

Winter et al. (1993) estimated the resting metabolic rate (RMR) of G. soricina to be $0.132 \mathrm{~W}$ for a $0.0109 \mathrm{~kg}$ bat. This resting rate, combining the alert resting rate between bouts of flight during the night (with the bat in the absorptive condition) and the daytime resting rate (in the post-absorptive condition), should be somewhat higher than BMR. McNab's (1988) overall regression equation for basal metabolic rate for bats, recalculated in SI units as $\mathrm{BMR}=2.63 M^{0.72}$ (in watts) (U. M. Norberg and T. H. Kunz, unpublished results), gives a BMR of $0.099 \mathrm{~W}$ for a bat with a body mass of $0.0105 \mathrm{~kg}$ and a BMR of $0.102 \mathrm{~W}$ for a bat with a body mass of $0.0109 \mathrm{~kg}$. McNab's empirical value for a $0.0096 \mathrm{~kg}$ G. soricina is $0.121 \mathrm{~W}$, which is higher than the value predicted from his regression for all bats $(0.093 \mathrm{~W})$. Using the slope of this regression, but with the $y$ intercept adjusted for G. soricina, the BMR would become $0.129 \mathrm{~W}$ for a $0.0105 \mathrm{~kg}$ bat and $0.132 \mathrm{~W}$ for a $0.0109 \mathrm{~kg}$ bat, the latter being the same as the estimate of RMR for a $0.0109 \mathrm{~kg}$ bat obtained by Winter et al. (1993). Using the regression through McNab's empirical value yields a BMR of $0.129 \mathrm{~W}$ for our $0.0105 \mathrm{~kg}$ hovering bat. Thus, the total metabolic power (power input; equation 1) for our hovering bat (mass $0.0105 \mathrm{~kg}$ ) becomes:

$$
P_{\text {methov }}=1.10[(0.340 / \eta)+0.129] \mathrm{W} .
$$

\section{Power required for horizontal flight}

The estimated power components for horizontal forward flight in G. soricina with a body mass of $0.0117 \mathrm{~kg}$ are given in Table 2 .

\section{Induced power}

We used equation 8 to estimate induced power in the bat flying at $4.2 \mathrm{~ms}^{-1}$, and $k$ was set to 1.56 , as obtained from the experiments with the hot-wire anemometer in which we measured induced velocity in hovering flight. This gives $P_{\text {ind }}=0.040 \mathrm{~W}$. 

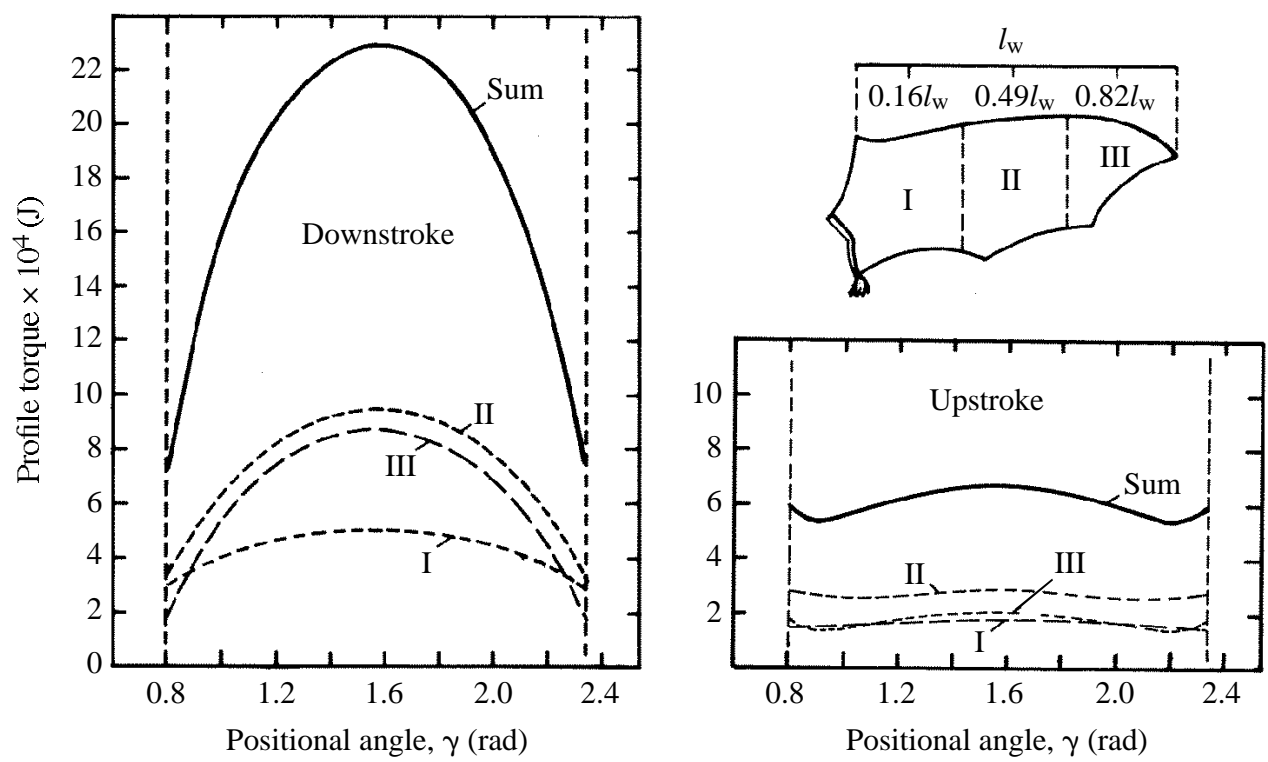

Fig. 5. The variation of profile torque (profile drag $\times$ moment arm) of one wing in a complete wing stroke of Glossophaga soricina during horizontal flight at $4.2 \mathrm{~ms}^{-1}$. The forces and velocities were calculated at radii (moment arms) $0.16 l_{\mathrm{w}}, 0.49 l_{\mathrm{w}}$ and $0.82 l_{\mathrm{w}}$ of strips I, II and III, respectively, of the wing (right-hand top figure), where $l_{\mathrm{W}}$ is wing length. The area below the summed curves in the downstroke (left-hand figure) and upstroke (right-hand bottom figure) make up the profile work of one wing, and the profile power for two wings is twice this amount times the wingbeat frequency.

\section{Profile power}

Fig. 5 shows the profile torque for the three wing strips and the summed curves for the downstroke and upstroke for one wing as functions of the positional angle $\gamma$ of the wings. During the upstroke the wings are flexed, but mostly at the wing base, while the hand wing is kept almost straight. The area of the outer strip (no. III) is thus the same during the upstroke as during the downstroke, whereas the area of the middle strip (no. II) decreases by approximately $10 \%$ and that of the proximal strip (no. I) by approximately $50 \%$. The area below the summed curves for the downstroke and upstroke is the profile work $(0.0023 \mathrm{~J}$, of which $0.0018 \mathrm{~J}$ is due to the downstroke and $0.00059 \mathrm{~J}$ to the upstroke). The summed profile power, $P_{\text {pro, }}$ is twice this work (for two wings) times the wingbeat frequency, $f_{\mathrm{w}}$, and is $0.055 \mathrm{~W}$.

\section{Parasite power}

In horizontal flight, the body was untilted, so the body frontal area, $S_{\mathrm{b}}$, becomes $0.00039 \mathrm{~m}^{2}$. At a forward speed of $4.2 \mathrm{~ms}^{-1}$ the parasite power, $P_{\mathrm{par}}$, is $0.0068 \mathrm{~W}$.

\section{Inertial power}

Fig. 6 shows the wings' inertial torque (bending moment) as a function of the positional angle, $\gamma$, of the wings. The inertial work is $0.00389 \mathrm{~J}$ during the downstroke and 


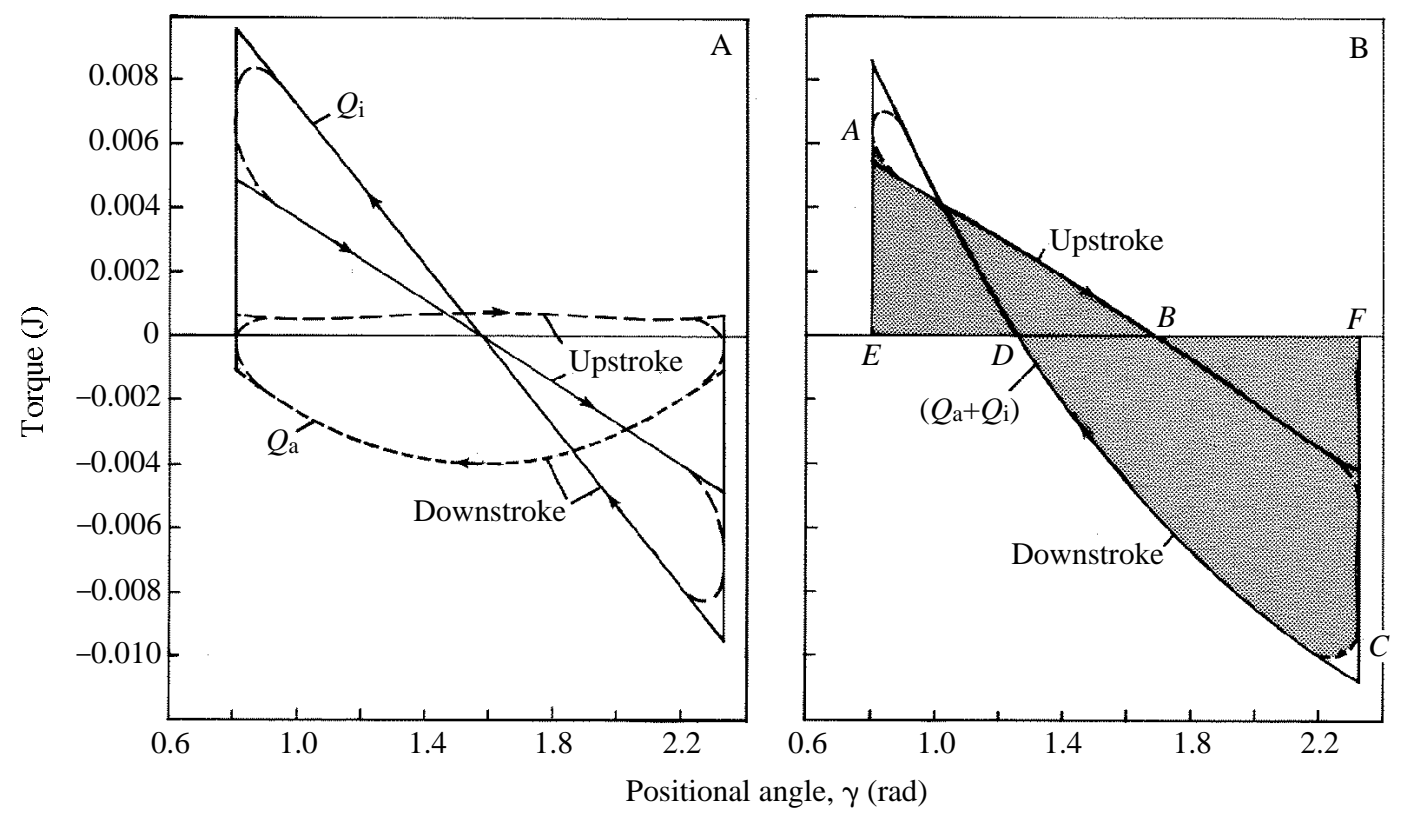

Fig. 6. Work diagram of Glossophaga soricina during horizontal forward flight at $4.2 \mathrm{~ms}^{-1}$. (A) The variation of the aerodynamic torque $\left(Q_{\mathrm{a}}\right)$ and inertial torque $\left(Q_{\mathrm{i}}\right)$ during a complete wing stroke (both wings) when the wingbeat frequency is $11.76 \mathrm{~s}^{-1}$. (B) The summed torque $\left(Q_{\mathrm{a}}+Q_{\mathrm{i}}\right)$, where area $E A B$ is the positive muscular work during an upstroke and area $D F C$ is that of a downstroke, which together make up the true expense. Of this, area $E A D$ represents the inertial work that is wasted, whereas area $D B C$ is the inertial power that is converted into useful aerodynamic work. See text and legend to Fig. 4 for further explanation.

$0.00194 \mathrm{~J}$ during the upstroke. The total inertial power, $P_{\text {iner, }}$, then is $f_{\mathrm{w}}$ times this work, and is $0.0686 \mathrm{~W}$. Using the procedure in Weis-Fogh (1972) for hovering flight, $61 \%$ of the inertial power was estimated to be converted into useful aerodynamic power. The true inertial power then becomes $P_{\text {iner,true }}=0.042 \mathrm{~W}$.

The sum of the aerodynamic and inertial power required for forward horizontal flight at $4.2 \mathrm{~ms}^{-1}$ is $P_{\text {hor }}=P_{\text {ind }}+P_{\text {pro }}+P_{\text {par }}+P_{\text {iner,true }}=0.144 \mathrm{~W}$. Using McNab's (1988) regression for bats, with the $y$-intercept adjusted to his empirical value for $G$. soricina (see above), BMR is $0.139 \mathrm{~W}$ for a $0.0117 \mathrm{~kg}$ bat. The total metabolic power for horizontal forward flight then becomes:

$$
P_{\text {methor }}=1.10[(0.144 / \eta)+0.139] \mathrm{W} \text {. }
$$

\section{Discussion}

Rayner and Thomas (1991) have shown that solid walls can have a significant effect on the flow around an animal flying in a confined space, which will result in a reduction in the induced downwash and thus in the induced power and induced power factor. The greatest effect is that of a solid ground plane (ground effect), which would cause a recirculation of the downwardly induced flow within the cage. Rayner and Thomas 
showed that, in a cubic confined volume with solid walls with sides of about the length of four wing spans, the induced power would be reduced by about $10 \%$ compared with hovering in the field (free space). In our investigation, however, the bottom of the chamber was made of netting that let air through, and the solid ground floor was approximately six wing spans $(1.5 \mathrm{~m})$ from the hovering bat. Any distortion of the momentum jet below the bat may, therefore, have been much less than in the example above (10\%). Still, the ceiling and walls may have had some influence on the downward flow, resulting in some savings of the induced power. Rayner and Thomas (1991) further showed that the reduction is relatively modest in fast forward flight. This minor discrepancy in the calculation of induced power from the condition in the field is not important for this investigation, since we are comparing different methods of estimating flight costs, under uniform conditions. It is, however, important to note that hovering in free space may cost slightly more than our estimated cost.

The speed observed during horizontal flight in G. soricina $\left(4.2 \mathrm{~ms}^{-1}\right)$ lies close to the minimum power speed $\left(V_{\mathrm{mp}}\right)$ according to the theoretical models (based on aerodynamic power only) of Rayner (1990; see also Norberg and Rayner, 1987) and Pennycuick (1989). According to Rayner's model, $V_{\mathrm{mp}}$ in a bat the size of $G$. soricina (body mass $0.0117 \mathrm{~kg})$ would be $3.9 \mathrm{~ms}^{-1}$ and the maximum range speed $\left(V_{\mathrm{mr}}\right)$ would be $5.0 \mathrm{~ms}^{-1}$; according to Pennycuick's model (Program 1A), $V_{\mathrm{mp}}$ is $4.7 \mathrm{~ms}^{-1}$ and $V_{\mathrm{mr}}$ is $7.5 \mathrm{~ms}^{-1}$. BMR is not included in these calculations. The inclusion of BMR causes an increase in $V_{\mathrm{mr}}$ (to $8.3 \mathrm{~ms}^{-1}$ with Pennycuick's model), but no change in $V_{\mathrm{mp}}$. By including inertial power in their models, $V_{\mathrm{mp}}$ would also become somewhat higher, and the range between $V_{\mathrm{mp}}$ and $V_{\mathrm{mr}}$ would become smaller, since the inertial power decreases with increasing speed. The flight speed recorded for G. soricina in the flight cage may therefore be rather close to the bat's $V_{\mathrm{mp}}$. A flight speed of about $7 \mathrm{~ms}^{-1}$ has been recorded in $G$. soricina flying in a $33 \mathrm{~m}$ long flight tunnel (Y. Winter, in preparation).

According to aerodynamic theory (equations 6-10) the mechanical power required for horizontal forward flight at $4.2 \mathrm{~ms}^{-1}$ for the $0.0105 \mathrm{~kg}$ bat should be approximately $10 \%$ lower (about $0.130 \mathrm{~W}$ ) than for the $0.0117 \mathrm{~kg}$ bat $(0.144 \mathrm{~W})$. The power required for hovering for a $0.0105 \mathrm{~kg}$ bat $(0.340 \mathrm{~W})$ would thus become approximately 2.6 times as large as the power required for forward flight at $4.2 \mathrm{~ms}^{-1}$ for the same bat. This difference is approximately $40-50 \%$ and $15-25 \%$ larger than those obtained with Pennycuick's $(1975,1989)$ and Rayner's $(1979 a, b, c)$ theoretical models, respectively. However, the inertial power is not included in their models, which means that they assumed perfect elastic storage of inertial work. In our experiments, the aerodynamic power required for hovering is about 1.5 times the aerodynamic power required for horizontal flight close to the minimum power speed, whereas the true inertial power required for hovering is 4.5 times that required for horizontal flight. Inertial power forms a larger proportion of the total power for hovering than for forward flight in our study and therefore makes up this larger difference (2.6-fold) in total power between hovering and forward flight.

In hovering flight, the true inertial power ( $\left.P_{\text {iner,true }}\right)$ in $G$. soricina constitutes $55 \%$ of the total power, and it is $22 \%$ higher than the aerodynamic power $\left(P_{\text {ind }}+P_{\text {pro }}\right)$. In medium speed and fast flights the true inertial power is lower; at $4.2 \mathrm{~ms}^{-1}$ it makes up $29 \%$ of the 
total power and it is $59 \%$ lower than the aerodynamic power $\left(P_{\text {ind }}+P_{\text {pro }}+P_{\text {par }}\right)$. WeisFogh (1972) estimated the overall mechanical power output in a hovering hummingbird (A. fimbriata, $0.005 \mathrm{~kg}$ ) to be $0.230 \mathrm{~W}$, of which $43 \%(0.098 \mathrm{~W})$ was due to wing inertia (true inertial power), which is $12 \%$ less than our result for G. soricina.

The power output for horizontal flight of a $0.0117 \mathrm{~kg} \mathrm{G}$. soricina would be $0.083 \mathrm{~W}$ for minimum power flight and $0.104 \mathrm{~W}$ for maximum range flight according to Pennycuick's model (1989), which includes an induced factor of $k=1.2$. According to Rayner's regression (Norberg and Rayner, 1987; Rayner, 1990), it would be $P_{\mathrm{mp}}=0.065 \mathrm{~W}$ and $P_{\mathrm{mr}}=0.078 \mathrm{~W}$. Inserting our empirically determined value $k=1.56$ in Pennycuick's model would increase $V_{\mathrm{mp}}$ to $5.0 \mathrm{~ms}^{-1}$ and $V_{\mathrm{mr}}$ to $8.0 \mathrm{~ms}^{-1}$, and the power outputs would become $P_{\mathrm{mp}}=0.101 \mathrm{~W}$ and $P_{\mathrm{mr}}=0.126 \mathrm{~W}$. All these values should be compared with the estimated aerodynamic power in $G$. soricina $\left(P_{\mathrm{ind}}+P_{\mathrm{pro}}+P_{\mathrm{par}}\right)$ of $0.102 \mathrm{~W}$, which is approximately the same as the corrected $P_{\mathrm{mp}}$ value $(0.101 \mathrm{~W})$ obtained with Pennycuick's model, which did not include inertial power.

We obtained the metabolic cost of horizontal flight in G. soricina by estimating the energy content of the nectar intake, giving $P_{\text {met,hor }}=1.63 \mathrm{~W}$ (Winter et al. 1993) for a $0.0117 \mathrm{~kg}$ bat, which is about $12 \times \mathrm{BMR}$. The average flight speed for this bat was $3.1 \mathrm{~ms}^{-1}$, which is lower than the speed used by the bat for which the power output was estimated and is probably also lower than the minimum power speed. Insertion of $P_{\text {methor }}=1.63 \mathrm{~W}$ into equation 12 gives a mechanical efficiency, $\eta$, of 0.11 . Compensating for the difference in flight speeds at which the metabolic and mechanical costs were estimated, the mechanical efficiency would become slightly larger and the multiple of BMR slightly smaller, because the metabolic cost would be larger for a bat flying at $3.1 \mathrm{~ms}^{-1}$ (if this is less than $V_{\mathrm{mp}}$ ) than for one flying at $4.2 \mathrm{~ms}^{-1}$ (if this is close to $V_{\mathrm{mp}}$ ). However, if the two speeds lie equidistant from $V_{\mathrm{mp}}$, they are comparable as regards the flight cost.

We also derived a value of approximately $2.92 \mathrm{~W}$ for a $0.0117 \mathrm{~kg}$ G. soricina for the metabolic power required for hovering flight from the nectar intake experiments (Winter et al. 1993). However, more data are needed before we can rely on this value. This estimate of hovering cost is about 1.8 times the metabolic power $(1.63 \mathrm{~W}$; Winter et al. 1993) required for horizontal flight at $3.1 \mathrm{~ms}^{-1}$ (which is below $V_{\mathrm{mp}}$ ). Theoretically, the metabolic power required for hovering flight for a $0.0105 \mathrm{~g}$ bat would be about $8 \%$ lower than that for a $0.0117 \mathrm{~kg}$ bat, and thus would be about $2.68 \mathrm{~W}$. Insertion of $P_{\text {methov }}=2.68 \mathrm{~W}$ into equation 11 gives a mechanical efficiency of $\eta=0.15$, and the metabolic power required to hover would be about $21 \times$ BMR.

Mechanical efficiency has been measured in only two bat species in horizontal flight and was found to range from 0.12 to 0.14 in Phyllostomus hastatus $(M 0.093 \mathrm{~kg})$ and from 0.22 to 0.31 for Pteropus alecto $(M 0.81 \mathrm{~kg}$ ) (Thomas, 1975). There is reason to believe that $\eta$ decreases with decreasing body mass in bats, as has been found for locomotion in other mammals (Heglund et al. 1982). Comparison between empirical estimates of metabolic power required for flight in bats, according to the regression equation in U. M. Norberg and T. H. Kunz (unpublished results), and theoretical estimates of mechanical power, according to Rayner's (1990) and Pennycuick's (1989) models, suggests that the mechanical efficiency should be about $0.05-0.08$ for a $0.0117 \mathrm{~kg}$ bat flying at minimum 
Table 3. Estimated values of the mechanical power $(\mathrm{P})$ required for hovering and horizontal flight (at $4.2 \mathrm{~ms}^{-1}$ ) and the corresponding mechanical efficiencies $(\eta)$ of the nectar-feeding bat Glossophaga soricina of body mass $0.0105 \mathrm{~kg}$ and $0.0117 \mathrm{~kg}$, respectively, based on different assumptions (see text for explanation)

\begin{tabular}{|c|c|c|c|c|}
\hline \multirow[b]{2}{*}{ Assumption } & \multicolumn{2}{|c|}{ Hovering } & \multicolumn{2}{|c|}{ Forward flight } \\
\hline & $P$ & $\eta$ & $P$ & $\eta$ \\
\hline Perfect elastic storage & $0.15 \mathrm{~W}$ & 0.006 & $0.10 \mathrm{~W}$ & 0.08 \\
\hline Zero elastic storage & $0.34 \mathrm{~W}$ & 0.15 & $0.14 \mathrm{~W}$ & 0.11 \\
\hline $\begin{array}{l}\text { Zero elastic storage with } \\
\text { wing virtual mass included }\end{array}$ & $0.43 \mathrm{~W}$ & 0.19 & $0.18 \mathrm{~W}$ & 0.13 \\
\hline
\end{tabular}

power speed. This should be compared with 0.076 obtained for $G$. soricina, if we assume perfect elastic storage (Table 3).

Table 3 shows the estimated minimum and maximum values of the mechanical power required to hover and fly horizontally at $4.2 \mathrm{~ms}^{-1}$ in G. soricina. If we ignore wing inertia as a cost (assuming perfect elastic storage), the mechanical power required to hover would become $P_{\text {hov }}=0.15 \mathrm{~W}$ and thatto fly horizontally would become $0.10 \mathrm{~W}$, which can be regarded as minimum values. Assuming zero elastic storage, the flight costs become $P_{\text {hov }}=0.34 \mathrm{~W}$ and $P_{\text {hor }}=0.14 \mathrm{~W}$, which are discussed above as the most reasonable values. If viscous forces are important when the wings accelerate, the inertia of the wings may be increased by the mass of air moving along them. Wing virtual mass would add about $47 \%$ to the wing mass, with the same proportional increase in inertial power. The flight powers would then become $P_{\text {hov }}=0.43 \mathrm{~W}$ and $P_{\text {hor }}=0.18 \mathrm{~W}$, which can be regarded as maximum values. If we compare these values with our values for the metabolic power, the corresponding ranges for the mechanical efficiency would become $\eta=0.066-0.19$ for hovering flight and $\eta=0.076-0.13$ for horizontal forward flight (Table 3).

We are indepted to $\mathrm{M}$. Tschapka for invaluable assistance during the entire investigation, to R. Kittman for the loan of his hot-wire anemometer and to F. Durst for its calibration. We further thank H. J. D. N. Aldridge, R. A. Norberg and two anonymous reviewers for valuable comments on the manuscript. This work was supported by grants from the Swedish Natural Science Research Council (B-GF 4455-302) to U.M.N. and from the Deutsche Forschungsgemeinschaft (He-1180/6-1) to O.v.H.

\section{References}

Anthony, E. L. P. And Kunz, T. H. (1977). Feeding strategies of the little brown bat, Myotis lucifugus, in southern New Hampshire. Ecology 58, 775-786.

Bernstein, M. H., Thomas, S. P. And Schmidt-Nielson, K.(1973). Power input during flight of the fish crow, Corvus ossifragus. J. exp. Biol. 58, 401-410.

CARPEnter, R. E. (1975). Flight metabolism of flying foxes. In Swimming and Flying in Nature (ed. T. Y.-T. Wu, C. J. Brokaw and C. Brennen), pp. 883-890. New York: Plenum Press.

CARPENTER, R. E. (1985). Flight physiology of flying foxes. J. exp. Biol. 114, 619-647.

CARPENTER, R. E. (1986). Flight physiology of intermediate-sized fruit bats (Pteropodidae). J. exp. Biol. 120, 79-103. 
Ellington, C. P. (1984a). The aerodynamics of hovering insect flight. II. Morphological parameters. Phil. Trans. R. Soc. Lond. B 305, 17-40.

Ellington, C. P. (1984b). The aerodynamics of hovering insect flight. V. A vortex theory. Phil. Trans. R. Soc. Lond. B 305, 115-144.

ELLINGTON, C. P. (1984c). The aerodynamics of hovering insect flight. VI. Lift and power requirements. Phil. Trans. R. Soc. Lond. B 305, 145-181.

Heglund, N. C., Fedak, M. A., Taylor, C. R. and Cavagna, G. A. (1982). Energetics and mechanics of terrestrial locomotion. IV. Total mechanical energy changes as a function of speed and body size in birds and mammals. J. exp. Biol. 97, 57-66.

Hudson, D. M. And Bernstein, M. H. (1983). Gas exchange and energy cost of flight in the whitenecked raven, Corvus cryrptoleucus. J. exp. Biol. 103, 121-130.

Hughes, P. M. And Rayner, J. M. V. (1991). Addition of artificial loads to long-eared bats Plecotus auritus: handicapping flight performance. J. exp. Biol. 161, 285-298.

KunZ, T. H. (1974). Feeding ecology of a temperate insectivorous bat (Myotis velifer). Ecology 55, 693-711.

KunZ, T. H. AND NAGY, K. A.(1988). Methods of energy budget analysis. In Ecological and Behavioral Methods for the Study of Bats (ed. T. H. Kunz), pp. 277-302. Washington, DC: Smithsonian Institution Press.

Margaria, R. (1968). Positive and negative work performances and their efficiencies in human locomotion. Int. Z. angew. Physiol. einschl. Arbeitsphysiol. 25, 339-351.

MASMAN, D. AND KLAASSEN, M.(1987). Energy expenditure during free flight in trained and free-living Eurasian kestrels (Falco tinnunculus). Auk 104, 603-616.

MCNAB, B. K. (1988). Complications inherent in scaling the basal rate of metabolism in mammals. Q. Rev. Biol. 63, 25-54.

NAGY, K. A. (1989). Field metabolic rate and food requirement scaling in mammals and birds. Ecol. Monogr. 57, 111-128.

Norberg, U. M.(1976a). Some advanced flight manoeuvres of bats. J. exp. Biol. 64, 489-495.

Norberg, U. M. (1976b). Aerodynamics, kinematics and energetics of horizontal flapping flight in the long-eared bat Plecotus auritus. J. exp. Biol. 65, 179-212.

NoRBERG, U. M.(1976c). Aerodynamics of hovering flight in the long-eared bat Plecotus auritus. J. exp. Biol. 65, 459-470.

NORBERG, U. M.(1990). Vertebrate Flight. Berlin: Springer-Verlag.

Norberg, U. M. AND RAYNER, J. M. V. (1987). Ecological morphology and flight in bats (Mammalia; Chiroptera): wing adaptations, flight performance, foraging strategy and echolocation. Phil. Trans. R. Soc. Lond. B 316, 335-427.

Pennycuick, C. J. (1968). Power requirements for horizontal flight in the pigeon. J. exp. Biol. 49, 527-555.

PennyCuick, C. J. (1975). Mechanics of flight. In Avian Biology (ed. D. S. Farner and J. R. King), pp. 1-75. New York: Academic Press.

PenNyCuick, C. J. (1989). Bird Flight Performance: A Practical Calculation Manual. Oxford: Oxford University Press.

Pennycuick, C. J., Heine, C. E., KirkPatrick, S. J. And Fuller, M. R. (1992). The profile drag of a hawk's wing, measured by wake sampling in a wind tunnel. J. exp. Biol. 165, 1-19.

PenNyCuick, C. J. AND Lock, A.(1976). Elastic energy storage in primary feather shafts. J. exp. Biol. 64, 677-689.

Pennycuick, C. J., Obrecht III, H. H. AND Fuller, M. R. (1988). Empirical estimates of body drag of large waterfowl and raptors. J. exp. Biol. 135, 253-264.

RAYNER, J. M. V. (1979a). A vortex theory of animal flight. I. The vortex wake of a hovering animal. J. Fluid Mech. 91, 697-730.

RAYNeR, J. M. V. (1979b). A vortex theory of animal flight. II. The forward flight of birds. J. Fluid Mech. 91, 731-763.

RAYNER, J. M. V.(1979c). A new approach to animal flight mechanics. J. exp. Biol. 80, 17-54.

RAYNER, J. M. V. (1990). The mechanics of flight and bird migration performance. In Bird Migration (ed. E. Gwinner), pp. 283-299. Berlin, Heidelberg: Springer-Verlag.

RAYNER, J. M. V. AND AlDRIDGE, H. D. J. N. (1985). Three-dimensional reconstruction of animal flight paths and the turning flight of microchiropteran bats. J. exp. Biol. 118, 47-265. 
RAyner, J. M. V. AND Thomas, A. L. R. (1991). On the vortex wake of an animal flying in a confined volume. Phil. Trans. R., Soc. Lond. B 334, 107-117.

Thollesson, M. And Norberg, U. M. (1991). Moments of inertia of bat wings and body. J. exp. Biol. 158, 19-35.

Thomas, S. P. (1975). Metabolism during flight in two species of bats, Phyllostomus hastatus and Pteropus gouldii. J. exp. Biol. 63, 273-293.

TUCKER, V. A. (1968). Respiratory exchange and evaporative water loss in the flying budgerigar. J. exp. Biol. 48, 67-87.

Tucker, V. A. (1972). Metabolism during flight in the laughing gull, Larus atricilla. Am. J. Physiol. 222, 237-245.

TUCKER, V. A. (1973). Bird metabolism during flight: evaluation of a theory. J. exp. Biol. 58, 689-709.

von Helversen, O. (1986). Blütenbesuch bei Blumenfledermäusen: Kinematik des Schwirrfluges und Energiebudget im Freiland. In Bat Flight - Fledermausflug (ed. W. Nachtigall), Biona Report 5, pp. 107-126. Stuttgart, New York: Gustav Fischer.

von HELVERSEN, O. AND REYER, H.-U.(1984). Nectar intake and energy expenditure in a flower visiting bat. Oecologia 63, 178-184.

WeBb, P. W. (1971). The swimming energetics of trout. II. Oxygen consumption and swimming efficiency. J. exp. Biol. 55, 521-540.

WeIs-Fogh, T. (1972). Energetics of hovering flight in hummingbirds and in Drosophila. J. exp. Biol. 56, 79-104.

WeIs-Fogh, T. (1973). Quick estimates of flight fitness in hovering animals, including novel mechanisms for lift production. J. exp. Biol. 59, 169-230.

Winter, Y., von Helversen, O., Norberg, U. M., Kunz, T. H. and Steffensen, J. F. (1993). Flight cost and economy of nectar feeding in the bat Glossophaga soricina (Phyllostomatidae: Glossophaginae). In Plant-Animal Interactions in Tropical Environments (ed. C. M. Naumann, W. Barthlott and K.-E. Schuchmann), pp. 167-174. Bonn: Museum Alexander Koenig. 